\title{
"Is IEEE VIS *that* good?" On key factors in the initial assessment of manuscript and venue quality
}

\author{
Nicholas Spyrison* \\ Monash University
}

\author{
Benjamin Lee ${ }^{\dagger}$ \\ Monash University
}

\author{
Lonni Besançon \\ Monash University
}

\begin{abstract}
Background:

Academic performance is at the heart of hiring decisions and funding applications. A commonly used performance metric is the prestige of venues which a researcher publishes scholarly articles in. This may influence researchers' individual perceptions of different venues, and subsequently cause them to discriminate research articles merely based on publication venue.
\end{abstract}

Objectives: $\quad$ We want to understand if this focus on prestige affects how visualization researchers behave when reading and citing articles in their own work, or if it is the case in administrative processes only (e.g., hiring, grants). We also want to understand how visualization researchers determine the prestige of any given venue.

Method: We ran an online survey open for 10 days that we sent out to visualization researchers.

Results: $\quad$ We gathered 46 responses through a sample of convenience. Unsurprisingly, publication venue plays the biggest part in how visualization researchers determine if they should read a given research article. Interestingly, when forming their opinion on said publication venues, rating systems and metrics are among the least important measures for quality.

Conclusion: We highlight the potential risks around focusing on venue when assessing research articles. We further underline the necessity to discuss with the community on strategies to switch the focus to robustness and reliability to foster better practices and less stressful publishing expectations.

Reproducibility: Data, materials and preregistration available on https://osf.io/ch6p4/

Index Terms: General literature-Metrics-Reliability-;

\section{INTRODUCTION}

Eligibility criteria to secure funding or positions in academia are almost always based on academic performance. Most of the metrics used are quantitative (e.g., h-index, number of publications, number of citations) [15], and others are a combination of quantitative and qualitative (e.g., the reputation of a journal and its impact factor) Young and early career researchers are often told to publish "high quality work in high quality venues", especially if they wish to pursue a career in academia.

\footnotetext{
*e-mail: nicholas.spyrison@monash.edu

†e-mail: benjamin.lee1@monash.edu

†e-mail: lonni.besancon@gmail.com
}

While this is arguably solid advice, there is usually an implied "as often as possible" followed after it. This (unrealistic) pressure to pursue both quality and quantity can result in an atmosphere of "publish or perish", which has been shown to be extremely detrimental to the mental health of researchers $[3,10,14,17]$ - particularly that of early career academics [19]. These high expectations can also diminish the trustworthiness, validity, and robustness of published scientific communications, as they can incentivize bad practices (e.g., p-hacking, HARK-ing [9], results fabrication) in order to cut corners and get published as soon as possible [5,10,13,15].

While the visualization community does not often rely on impact factor, we still tend to judge our conferences/journals based on their reputation or CORE ranking [1]. This results in venues effectively being ranked against each other, with some being more 'prestigious' than others. This notion of ranking was exemplified in a recent survey of researchers in human-computer interaction (which has ties to the visualization community) by Besançon et al. [6]. They found that while there was a willingness to experiment with alternative peerreview systems for smaller, less important venues, the community would not accept such drastic changes for the more highly regarded venues. This discrimination could highlight that the effects of venue prestige extend beyond simple grant writing and hiring decisions, with it affecting how we think, behave, and work as researchers. It is still unclear, however, if such findings translate to specifically the visualization community. We therefore set out to find out if the reputation and prestige of a venue is only relevant for administrative purposes (e.g., grant writing, hiring, organizational performance indicators), or if we as visualization researchers also take this into account when we read and subsequently cite works in our field.

To this end, we designed and sent out a survey to fellow researchers in the visualization community. Our survey aims at understanding how we, in the context of a literature search, decide whether or not to read any given article, and how important the publication venue is in this decision. In addition to this, our survey also aims to shed light on how visualization researchers assess the prestige and reputation of a venue. With this, we hope to gather insight on the importance of venue prestige and perhaps answer the question: "Is IEEE VIS really that good?"

\section{The SuRVEY}

In order to gauge the extent that visualization researchers are influenced by the prestige of a venue, we decided to frame our survey in the context of a literature search. Not only is this a very common activity, it is also one of the few situations where snap judgments on whether or not to read a paper in detail are made. That is, given limited information such as title, authors, and publication venue (i.e., metadata), which factor(s) contribute the most in determining if a paper should be read in detail or skipped altogether. Note that we specifically focus on how researchers read as opposed to cite papers. This difference is crucial as we assume that researchers will decide whether to cite a paper primarily based on its relevancy to their own work. In order to determine a paper's relevancy however, one will need to read it in the first place, hence the importance of examining factors in how we do these literature searches.

Our survey, inspired from the work of Soderberg et al. [20], revolves around three main questions: 


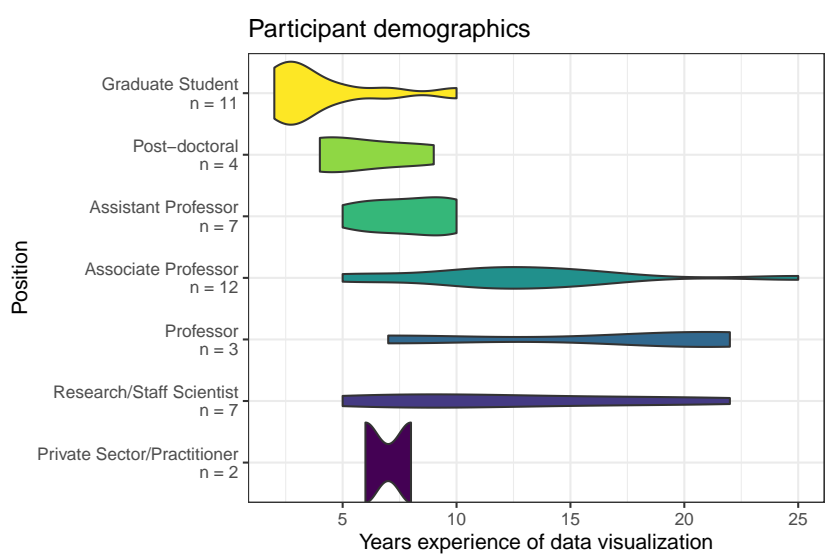

Figure 1: The demographics of our participants-violin plots of the distributions of years of data visualization experience within each level of position. Count within each position is also listed.

(1) How visualization researchers typically source articles when doing literature searches. Through this, we get an idea of the types of information that are generally used when making reading decisions. For example, a paper recommendation from a colleague has an implied endorsement which would not otherwise be there in a list of Google Scholar results.

(2) How important are certain factors in deciding whether to read an article in detail. In addition to its publication venue, we also chose to ask about the importance of other common factors, such as the researcher's personal familiarity with the authors, the authors' institution(s), the availability of data and research materials, and usage metrics (e.g., download and citation count).

(3) How researchers determine the prestige of a venue. Similar to above, we ask the importance of different factors in assessing the quality/prestige of a venue, such as its acceptance rate, opinions of fellow peers and mentors, and research metrics (e.g., Impact Factor, h5-index). This way we can get an idea if a conference like IEEE VIS is perceived as prestigious because the community says that it is, or because of some other more quantitative metric.

In total, the survey consisted of 21, 5-point-scale Likert items spread across three Likert scale questions. Each Likert scale had an extra slot to add an additional item to account for responses that we did not include. An optional free-form text field was included at the end of the survey to allow for additional comments. To keep the survey as succinct as possible, we collected only minimal demographic information, including only the survey respondent's position (i.e., professor, research scientist, post-doc, etc.) and reported years of experience in data visualization. The survey takes between 2 to 4 minutes to answer according to our pilot testing.

Survey respondents were required to have at least a Bachelor's degree, and needed to have been a (co-)author of at least one IEEE VIS paper submission. The pre-registration of our survey can be found at https://osf.io/jz2gr.

\section{Results from Planned Analyses}

In total, 46 researchers responded to the survey. We sourced respondents through a sample of convenience: reaching out to colleagues in various groups primarily via email and social media. $8.7 \%$ felt strongly enough to add additional comments in the free-form text at the end. $34.1 \%$ of the time an item was added to the Likert scale questions to evaluate against our default options. All analyses reported below are exploratory, and are conducted in bulk according to our preregistration. Compiled results can be found on our Github repository: https://github.com/nspyrison/citation_habits_ieee.
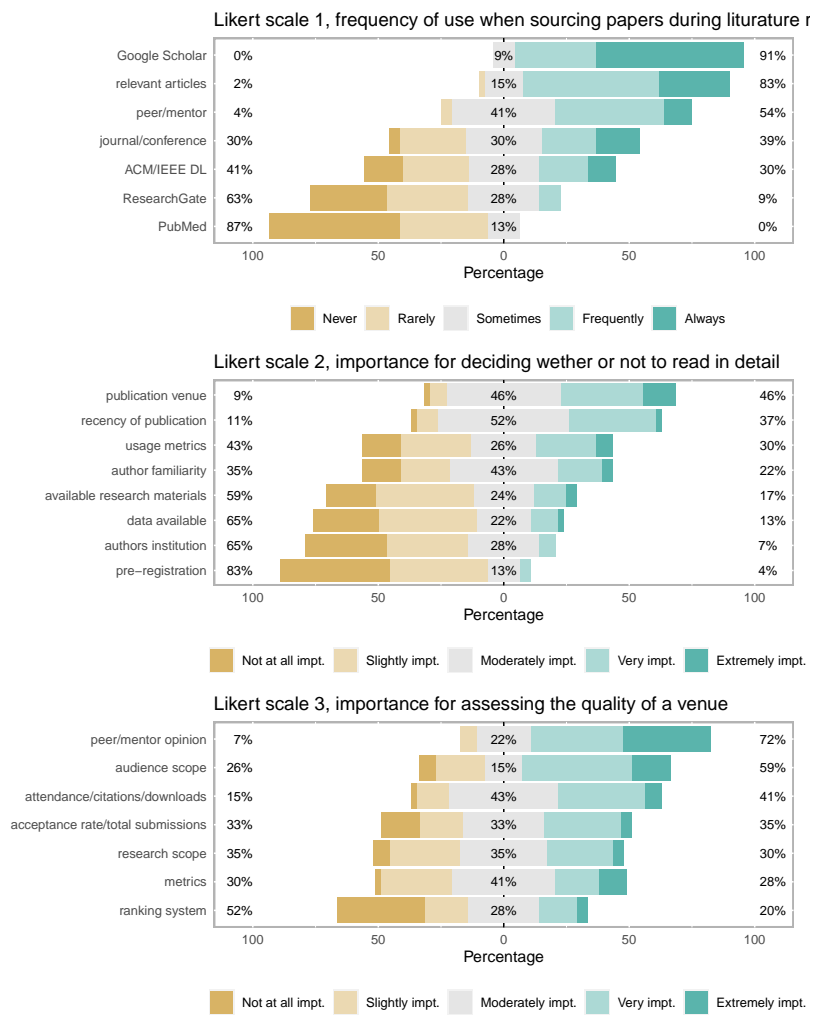

Figure 2: Likert plots of the 3 Likert scale questions, stacked percentile bar charts with aggregated percentages.

\subsection{Demographics}

The demographics of our survey respondents are in Fig. 1. There was considerable discussion on whether to use position or years of experience as the metric to compare across. We hold position as a better standard of comparison: it is less subjective, has less fringe cases, and does not have the issue of how much or how valuable a particular years' worth of experience is. We do note that position is still only an approximation of seniority in the field, but it will be the standard of comparison used in the rest of our analysis.

\subsection{Sourcing, reading, and estimating venue quality}

As described in Sect. 2, the bulk of our survey is split into three Likert scale questions: 1) how frequently different sources are used to find articles during literature searches; 2) how important different criteria are for deciding whether to read said articles in detail; and 3) how important different criteria are for assessing a venue's quality.

The Likert plots in Fig. 2 contain the responses to these questions. It comes with little surprise that Google Scholar enjoys a high frequency of use, even beating out citation trails (i.e., finding references mentioned in relevant articles). When it comes to deciding to read a paper in detail, two criteria particularly stand out: the publication venue, and how recently the article was published. These are followed by the usage metrics of the manuscript, and the familiarity that the respondent might have with the authors. Interestingly, the adoption of transparent research practices, such as making data and materials available or having a pre-registration, are among the criteria which matter the least. Lastly, the opinions of peers and mentors appears to be the most important factor in shaping our respondents' perception of a venue's quality. Perhaps most refreshing to see however is that quantitative metrics and ranking systems (e.g., CORE ranking [1]) are rated as being the least important. 


\begin{tabular}{|c|c|c|c|c|}
\hline \multicolumn{5}{|c|}{$\begin{array}{l}\text { Response of Likert item across position } \\
\text { Mean (standard deviation) }\end{array}$} \\
\hline \multirow[b]{2}{*}{ Likert item } & \multicolumn{4}{|c|}{ Position } \\
\hline & $\begin{array}{l}\text { Graduate Student, } \mathrm{n}= \\
\qquad 11\end{array}$ & $\begin{array}{l}\text { Assistant Professor, } \mathrm{n} \\
=7\end{array}$ & $\begin{array}{l}\text { Associate Professor, } \mathrm{n}= \\
12\end{array}$ & $\begin{aligned} & \text { Research/Staff Scientist, } \mathrm{n} \\
&=7\end{aligned}$ \\
\hline read author familiarity & $2.57(1.51)$ & $2.42(1.31)$ & $3.14(0.69)$ & $3.00(0.77)$ \\
\hline read authors institution & $2.14(1.07)$ & $1.75(0.87)$ & $2.29(0.76)$ & $2.45(1.04)$ \\
\hline read data available & $2.71(1.11)$ & $2.50(1.24)$ & $1.86(0.69)$ & $2.36(1.03)$ \\
\hline read pre-registration & $2.29(0.95)$ & $1.75(0.75)$ & $1.29(0.49)$ & $2.00(0.77)$ \\
\hline read publication venue & $3.29(0.95)$ & $3.08(1.00)$ & $3.57(0.53)$ & $3.73(0.90)$ \\
\hline read recency of publication & $3.43(0.79)$ & $3.17(0.72)$ & $3.29(0.76)$ & $3.55(0.69)$ \\
\hline read usage metrics & $3.00(1.29)$ & $2.58(1.08)$ & $3.00(1.29)$ & $3.45(1.13)$ \\
\hline source ACM/EEE DL & $2.86(1.46)$ & $2.67(1.23)$ & $3.00(1.15)$ & $3.00(1.48)$ \\
\hline source Google Scholar & $4.43(0.79)$ & $4.33(0.49)$ & $4.43(0.79)$ & $4.73(0.65)$ \\
\hline source journal/confrence & $2.71(0.95)$ & $3.42(1.24)$ & $2.71(1.11)$ & $3.36(1.36)$ \\
\hline source peer/mentor & $3.71(0.95)$ & $3.17(0.83)$ & $3.86(0.38)$ & $3.64(0.50)$ \\
\hline source PubMed & $1.43(0.53)$ & $2.17(0.72)$ & $1.57(0.53)$ & $1.27(0.65)$ \\
\hline source relevant articles & $3.86(0.90)$ & $4.17(0.39)$ & $4.29(0.76)$ & $4.18(0.87)$ \\
\hline source ResearchGate & $1.43(0.53)$ & $2.33(0.78)$ & $2.29(1.11)$ & $2.18(1.25)$ \\
\hline $\begin{array}{l}\text { venue acceptance rate/total } \\
\text { submissions }\end{array}$ & $3.14(1.21)$ & $2.83(1.19)$ & $3.14(0.90)$ & $2.91(1.14)$ \\
\hline venue audience scope & $4.00(1.00)$ & $3.00(1.35)$ & $3.86(0.69)$ & $3.36(1.12)$ \\
\hline venue metrics & $3.00(1.15)$ & $3.08(1.08)$ & $2.71(0.76)$ & $3.09(0.94)$ \\
\hline venue peer/mentor opinion & $3.57(0.98)$ & $3.92(1.08)$ & $4.43(0.53)$ & $4.18(0.98)$ \\
\hline venue ranking system & $2.29(1.25)$ & $2.17(1.11)$ & $2.57(1.27)$ & $2.18(1.40)$ \\
\hline venue research scope & $2.29(0.95)$ & $3.17(1.11)$ & $2.71(1.11)$ & $3.18(0.98)$ \\
\hline
\end{tabular}

Figure 3: Mean and standard deviation of the responses to Likert items by the participants' position.

\subsection{Differences across positions}

In our pre-registration, we had stated that we would "conduct a Spearman ranked correlation of the Likert scale responses in general and across experience levels", but the results are particularly difficult to interpret (see our GitHub repository for further discussion). We thus resort to the approach from Soderberg et al. [20]: reducing the distribution within each Likert item across the different positions in tabular form, and coloring cells based on the mean response. In large, there does not seem to be evidence that there is a difference in the distributions of Likert items across positions. We can see some slight variations in the results across positions. They remain modest however, and might be resulting noise in our relatively small sample.

\subsection{Quality of a venue and its publications}

We had included one Likert item in our survey that was not part of the above sections: "How correlated do you think the quality of a venue is with the quality of its research papers?". This was measured from 1 (no correlation) to 5 (strong positive correlation). We used this item as an example exploration to see how responses change across respondents' positions. Given the modest number of responses, we have quite a small number of observations within each position level and thus excluded some of levels with the fewest observations, specifically: private sector/practitioner $(n=2)$, professor $(n=3)$, and post-doc $(\mathrm{n}=4)$. The results, visible in Fig. 4, support respondents' belief of a strong relationship between the quality of a venue and the quality of its publications. Similar to the results presented in Sect. 3.3, there does not seem to be evidence of a difference across positions.

\subsection{Categorizing variation}

We created a mixed regression model, regressing on the response of all Likert items. We used position as it interacts with years of visualization experience and the topic of the Likert section as fixed variables, and measured the random effects of the participants and the individual Likert items. The form of our model is as follows:

$$
\widehat{Y}=\mu+\alpha_{i} * \beta_{j}+\mathbf{Z}+\mathbf{W}+\varepsilon \text {, where }
$$

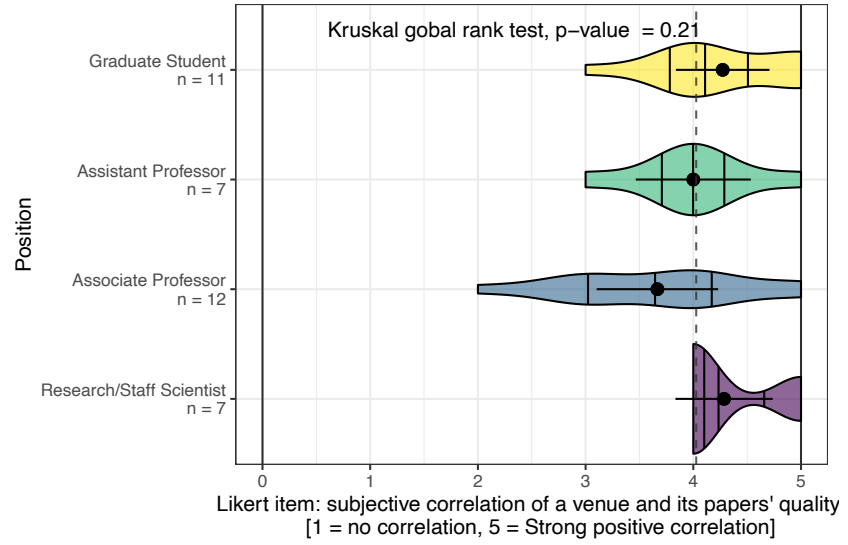

Figure 4: Violin plots illustrating the distributions of each position as they assess the correlation of the quality of a venue with that of its papers. A gray dashed line marks the global mean. Vertical lines mark the 25,50 , and 75 quantiles, circles show the mean, while horizontal lines show the $95 \%$ confidence intervals of the mean. The global Kruskal-Wallis rank test evaluates if there is a significant difference in any level, rather than for specific pairwise comparisons of levels.

$\mu$ is the intercept of the model including the mean of random effect $\alpha_{i}$ fixed term for position

$\beta_{j}$ fixed term for years of visualization experience

$\gamma_{k}$ fixed term for what the Likert question was about $\mid k \in$ (sourcing an article, detailed reading of it, assessing venue quality)

$\mathbf{Z}$ the random effect of the participant

$\mathbf{W}$ the random effect of the Likert item

$\varepsilon \sim \mathscr{N}(0, \sigma)$, the error term of the model.

The random effects of the participant and the Likert item explain an astonishing $44.9 \%$ of the overall variation in this subjective evaluation of correlation strength, while the fixed effects explained only $4.7 \%$ for a combined conditional $R^{2}$, explaining $49.6 \%$ of the variation in response of the variation. In terms of the magnitude of coefficients, continuous years of experience is almost perfectly independent of the Likert item response. Of the differences among position, the largest mean difference (on a 1 to 5 point Likert scale) was 0.62 (between graduate students and private sector/practitioners). The largest difference between Likert questions was 0.52 - between sourcing frequency and importance to read in detail.

In summary, the mixed model has sizable predictive power. The bulk of the variation explained in the model comes from the random effects of the Likert items and the participants rather than the fixed effects. This suggests that the location of the true population mean and respondents' interpersonal differences explain most of the variations in the responses more than position or years of experience, thereby confirming our previous interpretations. In addition to the preregistered analysis we also looked at factor analysis and at the text responses. These can be found in our GitHub repository.

\section{Discussion}

The motivating force behind this work was us wanting to find out if publishing at a prestigious conference like IEEE VIS is actually that important from a research perspective, as opposed to hiring, job, and/or administrative purposes.

The undeniably unshakable confidence in top venues may potentially be detrimental. From our survey, the mentality of favoring top venues when judging the worth of articles, at least at face value, appears to be pervasive. Students and early career researchers are typically trained with the mentality that they should strive to publish 
at top-tier venues in order to get citations and better job opportunities. It is therefore not surprising that this mindset also permeates to how we evaluate articles published by others. Pressure to publish in high-impact venues is likely to cause undue stress $[3,10,14,17]$, directly impacting the mental health and performance of researchersespecially of those earlier in their careers [19]. However, it is also not uncommon to turn around and publish at "lower tier" venues, particularly as a result of constant rejections and a desire to move on to other projects. This practice directly hinders researchers and the dissemination of knowledge: reformatting articles is time-consuming [16] and affects the timeliness of research. ${ }^{1}$ Ultimately, to mitigate these problems, we believe, all research should be judged based on its own merits regardless of venue. While this may seem impractical because of the time it would require, some institutions have already started to make significant change to their evaluation policies. Among those we can think of University of Ghent that decided to "step out of the rat race" by abandoning quantitative evaluations altogether in favor of a more holistic qualitative approach [18]. Particularly relevant to abandoning evaluation based on flawed metrics is the choice of more and more funding institutions to randomly allocate money to researchers in the hope that chance would "create more openness to ideas that are not in the mainstream" and therefore perhaps allow more breakthrough through more innovative out-of-the-box ideas [2]. Finally, while anecdotal, some courageous researchers removed venues from their list of publications on their academic CV in the hope that it will help foster a more quality-based evaluation of research articles [21,22]. This goes to show that despite how difficult/impractical we think alternatives might be, some solutions are already out there. They are of course not perfect, but neither is our current system, and science is an iterative process anyways, so we can start from there.

Influence of peers and mentors to shape researchers' perceptions. What we find fascinating from our results is the strong influence of our peers and mentors in shaping which venues we perceive as prestigious, in contrast to more "objective" measures such as h5-index and ranking systems. From Fig. 3, this mindset seems to be consistent across job positions. What this implies is that the visualization community, as human beings, is responsible for this favoritism towards certain venues and eventually probably sets some sort of self-fulfilling prophecy around venue quality. This is a good thing in some sense, as this empowers us as individuals to call into question how we differentiate between venues, and therefore have a measurable impact in changing this perception. On the other hand, it is likely that the root cause of these issues is institutional in nature, as the reason why your mentor, advisor, or your peers may care about prestige is because they are pressured into by their institution. It is encouraging however that based on our survey results, researchers personally do not find rating systems to be as important of a measure as the institutions and faculties that employ them. In this sense, the ability to sway the community's opinion rests on these people, as early career researchers would be unlikely to call into question what their superiors advise them to do. Whether or not there is a desire for such a paradigm shift has yet to be seen however.

Chasing prestige could incentivize misconduct. In order to secure positions or funding, researchers can feel pressured to publish at prestigious venues. Our results seem to highlight the pervasiveness of prestige in the visualization community. The pressure to pursue prestige may eventually push researchers to cut corners and could lead to questionable research practices. Indeed, previous work investigating the matter has found that the most prestigious journals (usually based on Impact Factor) often also have the highest retraction numbers [11]-largely as a result of fraud or error [12] and in some-part driven from more readers. Recent work even suggests that the reliability of scholarly communications might be inversely

${ }^{1}$ And one's chances of getting more citations, whatever that's worth. proportional to a journal's ranking [7]. It thus seems that the overreliance on the prestige of academic venues can wrongly incentivize misconduct and may hinder the reliability and trustworthiness of published scientific work, as researchers try harder and harder to get published in these venues. Since the infamous replication crisis in psychology, some research fields or institutions have tried to move away from the use of metrics, or to adopt practices that improve scientific rigor and transparency. While the visualization research community has not been shaken by a replication crisis (yet) or research misconduct (yet), one cannot rationally hope that this remains the status quo. In particular, our results highlight how current best practices for robust research results (such as transparency of research materials) are not strongly valued by the community. While visualization researchers might think that we are immune to issues such as a replication crisis, a recent study has warned about its possibility in empirical computer science [8] or in human-computer interaction [4]. While being optimistic about the quality of research in our field is certainly a good thing, we should act proactively to make sure that we will try to preserve the mental health of researchers and not incentivize questionable research practices. If the shift is already happening in other places and communities, we have no reason to wait.

Limitations and future work. As with any research endeavor, there are limitations in our survey. First, our convenience sampling of 46 survey respondents may be biased by us primarily inviting present and past colleagues, who likely would share similar research beliefs as compared to a sample of entirely different and varied institutions and/or countries. Second, there may be factors other than prestige when considering venue, such as its familiarity and recognizably (e.g., finding visualization research in biomedical sciences). And third, we focus on the gut reaction that researchers would have when deciding to read a paper or to skip it, particularly when given surface-level information. The effects of how we treat venues differently extends far beyond this however, affecting where we decide to send our papers for review, whether we cite a paper and draw inspiration from it, or even if we attend the conference in the first place. While we acknowledge these limitations (and there are likely more), we hope this is but a starting point of an interesting and fruitful discussion with fellow workshop participants. This might lead to drafting a position piece on how we could initiate a shift in the visualization community to become more open minded in how we perceive and evaluate manuscripts and venues.

\section{CONCLUSION}

This survey highlighted the human nature behind omnipresent prestige considerations for venues and manuscript. Visualization researchers subjective evaluation do not seem to place as much weighting in a venue's prestige as compared with the metric system that so impact their careers and future prospects. Nonetheless, the focus on venue prestige is inherently present in our considerations and might, in the long run, negatively impact the reliability of our research. Moving beyond these considerations and embracing robustness over prestige is achievable. As changes are more than unlikely to come from publishers, it is up to us, visualization researchers, to make sure that our focus stays or perhaps shifts towards more reliable research practices. Hopefully, this provocative piece will not increase the interest in publishing at top venue, but instead make people question why there is so much emphasis on venue and therefore help them initiate a much needed change.

\section{ACKNOWLEDGMENTS}

We want to thank in advance all the people that will be amazed and convinced by our provocative yet compelling manuscript and/or presentation and will help us transition to a system that will incentivize transparency and robustness while preserving the mental health of young academics. Oh, we also thank our survey respondents! 


\section{RefERENCES}

[1] Core computing research \& education conference portal. http:// portal. core.edu.au/conf-ranks/. Accessed: 2021-07-28.

[2] D. Adam. Science funders gamble on grant lotteries. Nature, 575(7785):574-5, 2019. doi: 10.1038/d41586-019-03572-7

[3] E. J. Andrews, S. Harper, T. Cashion, J. Palacios-Abrantes, J. Blythe, J. Daly, S. Eger, C. Hoover, N. Talloni-Alvarez, L. Teh, N. Bennett, G. Epstein, C. Knott, S. L. Newell, and C. K. Whitney. Supporting early career researchers: insights from interdisciplinary marine scientists. ICES Journal of Marine Science, 77(2):476-485, 01 2020. doi: 10. 1093/icesjms/fsz247

[4] L. Besançon and P. Dragicevic. The Continued Prevalence of Dichotomous Inferences at CHI. In CHI '19 - Proceedings of CHI Conference on Human Factors in Computing Systems Extended Abstracts, p. 1-11. ACM, Glasgow, United Kingdom, May 2019. doi: 10.1145/3290607. 3310432

[5] L. Besançon, N. Peiffer-Smadja, C. Segalas, H. Jiang, P. Masuzzo, C. Smout, E. Billy, M. Deforet, and C. Leyrat. Open science saves lives: lessons from the covid-19 pandemic. BMC Medical Research Methodology, 21(1):1-18, 2021. doi: 10.1186/s12874-021-01304-y

[6] L. Besançon, N. Rönnberg, J. Löwgren, J. P. Tennant, and M. Cooper. Open up: a survey on open and non-anonymized peer reviewing. $R e$ search Integrity and Peer Review, 5(1):1-11, 2020. doi: 10.1186/ s41073-020-00094-z

[7] B. Brembs. Prestigious science journals struggle to reach even average reliability. Frontiers in Human Neuroscience, 12:37, 2018. doi: 10 3389/fnhum.2018.00037

[8] A. Cockburn, P. Dragicevic, L. Besançon, and C. Gutwin. Threats of a replication crisis in empirical computer science. Commun. ACM, 63(8):70-79, July 2020. doi: $10.1145 / 3360311$

[9] A. Cockburn, C. Gutwin, and A. Dix. HARK No More: On the Preregistration of $\mathrm{CHI}$ Experiments, p. 1-12. Association for Computing Machinery, New York, NY, USA, 2018.

[10] M. A. Edwards and S. Roy. Academic research in the 21st century: Maintaining scientific integrity in a climate of perverse incentives and hypercompetition. Environmental engineering science, 34(1):51-61, 2017. doi: ttps://doi.org/10.1089/ees.2016.0223

[11] F. C. Fang, A. Casadevall, and R. P. Morrison. Retracted science and the retraction index. Infection and Immunity, 79(10):3855-3859, 2011. doi: 10.1128/IAI.05661-11

[12] F. C. Fang, R. G. Steen, and A. Casadevall. Misconduct accounts for the majority of retracted scientific publications. Proceedings of the National Academy of Sciences, 109(42):17028-17033, 2012. doi: 10. 1073/pnas. 1212247109

[13] M. R. Haley. On the inauspicious incentives of the scholar-level hindex: an economist's take on collusive and coercive citation. Applied Economics Letters, 24(2):85-89, 2017. doi: 10.1080/13504851.2016. 1164812

[14] R. Horne, K. J. Petrie, and S. Wessely. H-index pathology: implications for medical researchers and practitioners. BMJ, 339, 2009. doi: 10. 1136/bmj.b5356

[15] C. J. The darker side of quantitative academic performance metrics. South African Journal of Science, 115(7/8), Jul. 2019. doi: 10.17159/ sajs. 2019/5785

[16] A. G. LeBlanc, J. D. Barnes, T. J. Saunders, M. S. Tremblay, and J.-P. Chaput. Scientific sinkhole: The pernicious price of formatting. PLOS ONE, 14(9):1-7, 09 2019. doi: 10.1371/journal.pone.0223116

[17] G. O'Neill and M. Schroijen. Early-career researchers and mental health. Impact, 2018(12):44-45, 2018.

[18] E. Redden. Ghent university, in belgium, embraces new approach to faculty evaluation less focused on quantitative metrics, Jan 2019.

[19] E. N. Satinsky, T. Kimura, M. V. Kiang, R. Abebe, S. Cunningham, H. Lee, X. Lin, C. H. Liu, I. Rudan, S. Sen, et al. Systematic review and meta-analysis of depression, anxiety, and suicidal ideation among ph. d. students. Scientific Reports, 11(1):1-12, 2021. doi: 10.1038/ s41598-021-93687-7

[20] C. K. Soderberg, T. M. Errington, and B. A. Nosek. Credibility of preprints: an interdisciplinary survey of researchers. Royal Society Open Science, 7(10):201520. Publisher: Royal Society. doi: 10.1098/ rsos. 201520

[21] J. Tennant. Publication metrics. http://fossilsandshit.com/ research/metrics/.

[22] J. Tennant. Do you list journal names and/or impact factors on your cv? how about not doing this in 2018! use article-level citations and metrics instead, it's just common sense https://t.co/tiypwywcpe, Jan 2018. https://twitter.com/Protohedgehog/status/ $950312950857981952 ? \mathrm{~s}=20$. 